[NTERFFAET TECNOLOGGCA

\title{
A IMPLANTAÇÃO DO METODO 5S NO SETOR DE PROTOTIPOS EM UMA EMPRESA DO RAMO MOVELEIRO NO INTERIOR DE SÃO PAULO
}

\author{
THE IMPLEMENTATION OF THE 5S METHOD IN THE SECTOR OF PROTOTYPES \\ IN A COMPANY OF RAMO MOVELEIRO IN THE INTERIOR OF SÃO PAULO
}

\author{
Fabricio Dominguês Antonio - fabriciodantonio@ hotmail.com \\ Angelita Moutin Segoria Gasparotto - angelita.gasparotto@ fatectq.edu.br \\ Faculdade de Tecnologia de Taquaritinga (FATEC) - SP - Brasil
}

DOI: 10.31510/infa.v15i2.394

\begin{abstract}
RESUMO
Este artigo apresenta que um ambiente limpo e organizado são condições de extrema importância para alcançar alta qualidade na fabricação de produtos, e ter baixo custo para se manter em competitividade no mercado. Os conceitos do programa $5 \mathrm{~S}$ abordam esses quesitos e sustentam as empresas que buscam estas condições. O objetivo deste artigo é implantar o método $5 \mathrm{~S}$ no setor de industrialização de protótipos em uma empresa de médio porte do setor moveleiro. O método utilizado foi de pesquisa exploratória em uma empresa do setor moveleiro localizada no interior de São Paulo e com revisão bibliográfica. Por meio da implantação do Programa 5S a empresa apresentou resultados positivos através da análise de gráficos, tendo em vista o aumento na qualidade de produtos acabados com máxima utilização de espaço e aproveitamento de matéria-prima. Com isso a empresa visa a Certificação ISO 9001:2015, tendo como parâmetro o programa 5S (Seiri-utilização, Seiton-ordenação, Seisolimpeza, Seitsuke-saude, Shitsuke-autodiscilina) e para manutenção do programa utilizar o método PDCA (PLAN, DO, CHECK, ACTION). O resultado obtido se deu pela execução do conceito 5S no setor de protótipos, com mão obra qualificada, ambiente adequado direcionados pelos procedimentos de organização, ordenação, limpeza, preocupação com o bem-estar do colaborador e treinamentos que resultou em alcançar o estado desejado, como redução de custo, melhoria no processo, maior qualificação de funcionários e ambiente de trabalho organizado.
\end{abstract}

Palavras-chave: Programa 5S. Treinamento. ISO 9001:2015

\begin{abstract}
This article presents that a clean and organized environment are conditions of extreme importance to achieve high quality in the manufacture of products, and to have low cost to remain competitive in the market. The concepts of the $5 \mathrm{~S}$ program address these issues and support the companies that seek these conditions. The objective of this article is to implement the $5 \mathrm{~S}$ method in the sector of prototype industrialization in a medium-sized company in the furniture sector. The method used was exploratory research in a furniture company located in the interior of São Paulo and with a bibliographical review. Through the implementation of the 5S Program, the company presented positive results through graphic analysis, in view of
\end{abstract}


the increase in the quality of finished products with maximum use of space and use of raw material. With this, the company aims at ISO 9001: 2015 Certification, having as parameter the $5 \mathrm{~S}$ program (Seiri-utilization, Seiton-ordenação, Seiso-limpieza, Seitsuke-saude, Shitsukeautodiscilina) and for program maintenance to use the PDCA, DO, CHECK, ACTION). The result was achieved by the execution of the $5 \mathrm{~S}$ concept in the prototype sector, with skilled workmanship, adequate environment directed by the procedures of organization, ordering, cleaning, concern with the well-being of the employee and training that resulted in achieving the desired state, such as cost reduction, process improvement, higher qualification of employees and organized work environment.

Keywords: Program 5S. Training. ISO 9001: 2015

\section{INTRODUÇÃO}

O programa 5S surgiu no Japão após a Segunda Guerra Mundial com muita destruição e com poucos recursos naturais, o país buscou meios para continuar a produzir com eficiência e qualidade utilizando o mínimo dos recursos possíveis, o programa recebe esse nome devido as cinco palavras japonesas que se iniciaram com a letra S, os sensos: utilização (seiri), ordenação (seiton), limpeza (seiso), saúde (seiketsu) e disciplina (shitsuke). Para o sucesso do programa, é necessário um trabalho eficaz, onde todos os envolvidos entendam e se comprometam com a implementação. Através da aplicação destes cinco sensos, é possível melhorar a qualidade do ambiente de trabalho e a qualidade de vida dos colaboradores, construindo um ambiente produtivo e saudável (CAPERUCCI; SILVA; SANTOS, 2016).

Este artigo busca abordar os fundamentos, conceitos e benefícios da adoção do programa 5S na empresa moveleira, bem como, a possibilidade de corrigir falhas e desperdícios, mobilizando, motivando e conscientizando os colaboradores na busca pela gestão da qualidade possibilitando que a organização atinja os seus objetivos como produzir com qualidade e baixo custo em um ambiente organizado no setor de protótipos.

\section{REVISÃO DA LITERATURA E ESTUDO DE CASO}

Por meio desta revisão é possível entender sobre o assunto apresentado neste artigo, que aborda os conceitos fundamentais do programa $5 \mathrm{~S}$ no setor de protótipos de uma empresa moveleira.

Uma empresa de médio porte, no Brasil, segundo o IBGE, é caracterizada pela quantidade de funcionários que ela possui. Se for indústria, é considerada como média empresas com 100 a 499 empregados. 


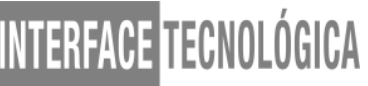

O estudo de caso é desenvolvido em empresa moveleira situada no interior de São Paulo que tem em torno de 350 colaboradores, com um parque fabril de $30000 \mathrm{~m}^{2}$, e com forte atuação em processos licitatórios, que atende a mobiliários escolares, aço e escritório. Os processos de licitações necessitam de amostras e/ou protótipos referentes aos descritivos técnicos abordados para ser apresentados em um curto período de tempo que variam de 3 a 30 dias dependendo do produto e da complexidade.

O artigo apresenta os problemas encontradas na empresa, a utilização do conceito e o objetivo alcançado.

\section{ARRANJO FISICO DO SETOR DE PROTÓTIPOS}

Arranjo físico é definido como um meio de transformação e como são posicionadas as várias tarefas de operação em que estão alocadas nestes recursos transformadores (SLACK; CHAMBERS; JOHNSTON, 2009).

Foi feito analisado que no setor de desenvolvimento os posicionamentos de maquinas estavam desorganizados e o fluxo de materiais não estavam seguindo um trajeto adequado ficando estocados em locais inapropriados, sendo assim foi apresentado um novo modelo de layout para o posicionamento de cada equipamento e estoques de matéria prima e produtos acabados como apresentado no exemplo:

Ilustração 1: Layout de como deve ficar o ambiente de trabalho

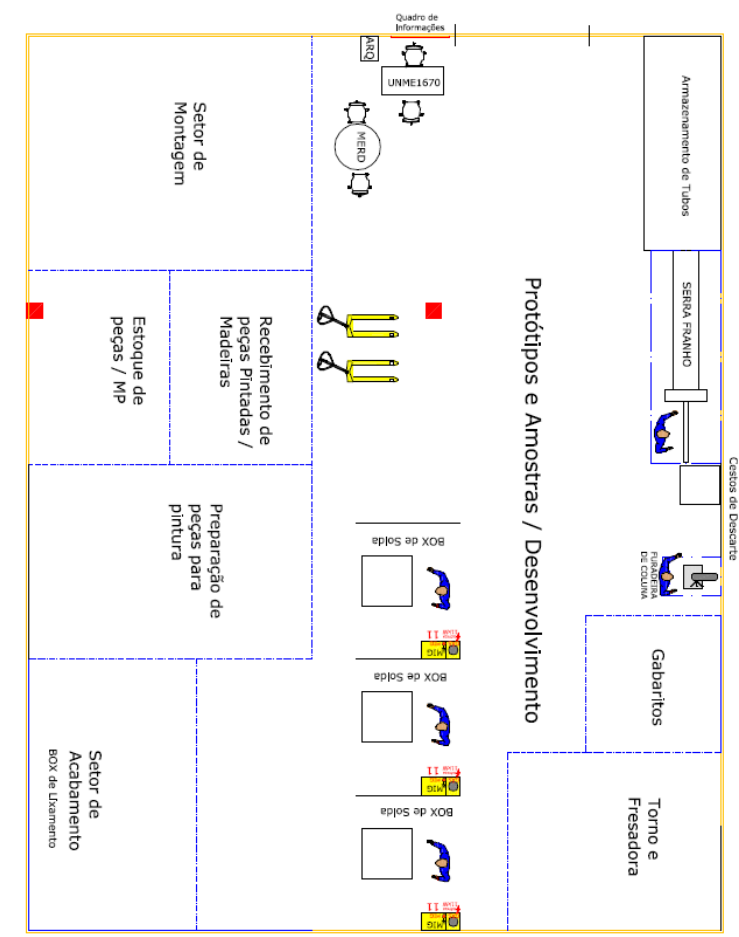

Fonte: Elaborado pelo autor (2018) 


\section{SIGNIFICADO DE CADA "SENSO"}

O senso de utilização é separar tudo aquilo que é útil e necessário daquilo que é inútil e desnecessário. Todo equipamento, maquinário e materiais terão sua destinação da forma correta direcionando cada coisa no seu lugar (ABRANTES, 2007).

Lima, Galan, Castilho, Oliveira (2018) definem que de tudo o que é útil e necessário seja eles maquinários, equipamentos e matérias devem ser classificados por ordem crescente de usabilidade, do mais acessível ao mais distante. E assim ter tudo a disposição conforme o uso de cada um e estando visível para que qualquer pessoal possa encontrar.

Costa et al. (2017) diz que o senso de limpeza é eliminar a sujeira para manter limpo o ambiente (parede, armários, o teto, gaveta, estante, piso). O mais importante neste conceito não é o ato de limpar e/ou eliminar a sujeira, mas o ato de "não sujar". Isto significa que além de limpar é preciso identificar a fonte de sujeira e as respectivas causas, de modo a podermos evitar que isto ocorra, mantendo um reconhecimento do ambiente, e sempre tendo que encarar como uma oportunidade de inspeção.

Caperucci, et al. (2016) compartilham que senso de higiene ou saúde significa ter condições que favoreçam à saúde física e mental. Garantir um ambiente com boas condições áreas sanitárias e comuns (lavatórios, banheiros...), zelar pela higiene pessoal e cuidar para que as informações e comunicados sejam claros, de fácil leitura e compreensão para todos aqueles que tiverem acesso ao ambiente. Significa ainda ter comportamento ético, promover um ambiente sem riscos à saúde, e ainda evitar que acidentes aconteçam.

Silva, (1994) diz que "a disciplina coroa a persistência da educação e treinamento" e com isso entendemos que devemos cumprir rigorosamente as normas, regras e procedimentos. Este senso é o resultado da prática do exercício dos $4 \mathrm{~S}$ anteriores pois mexe com a cultura e o comportamento das pessoas.

Segundo Ribeiro (2006) a disciplina é cumprir as normas de uma forma rigorosa que atinja todos da equipe.

\section{IMPLANTAÇÃO DO MÉTODO “5S”}

A empresa moveleira tem por finalidade fazer implementar o conceito do programa de qualidade 5S para melhorar e reorganizar o setor. 


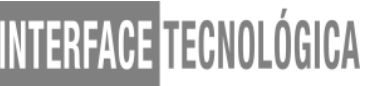

O Programa 5S é a sustentação para aplicação do programa de qualidade, e quando bem desenvolvido é reconhecido como uma excelente ferramenta de transformação na organização. (GONZALEZ, 2005)

Capeucci, et al. (2017) apresenta que o programa 5S foi desenvolvido pelo Prof ${ }^{o}$ Kaoro Ishikawa com o objetivo de combater o desperdício, com base nas origens japonesas dos sensos (seiri, seiton, seiketsu e shitsuke) que traduzido é utilização, organização, limpeza, saúde e autodisciplina.

Ishikawa (1993) diz que a qualidade total está em atender as necessidades do mercado com homogeneidade nos processos para fabricar e vender um produto mais barato produzindo satisfação ao consumidor final. Por meio disso a empresa moveleira tem a necessidade de garantir que seus produtos saiam com qualidade; portanto a empresa visa melhorar o ambiente de trabalho, processo e capacitação dos colaboradores utilizando o método $5 \mathrm{~S}$ de qualidade.

Os principais benefícios da metodologia 5S são:

1. Maior produtividade pela redução da perda de tempo procurando por objetos. Só ficam no ambiente os objetos necessários e ao alcance da mão

2. Redução de despesas e melhor aproveitamento de materiais. A acumulação excessiva de materiais estimula a desorganização.

3. Melhoria da qualidade de produtos e serviços

4. Redução de acidentes do trabalho

5. Maior satisfação das pessoas com o trabalho

\section{APLICAÇÃO DO CONCEITO DE QUALIDADE PROGRAMA "5S" NO SETOR DE DESENVOLVIMENTO DE PRODUTOS}

Para a aplicação do programa 5S, foi estabelecido por meio de um cronograma para implantação do conceito como mostrado na Tabela 1.

Tabela 1: Cronograma para implantação do método programa 5S.

\begin{tabular}{|c|c|c|c|c|c|c|c|c|}
\hline \multirow{2}{*}{ PROGRAMA 5S } & \multicolumn{7}{|c|}{ MÊS DE ABRIL/MAIO } & \multirow{2}{*}{ METAS } \\
\hline & D & $S$ & $\mathbf{T}$ & $\mathbf{Q}$ & $\mathbf{Q}$ & $S$ & $\mathbf{S}$ & \\
\hline \multirow{5}{*}{ SEIRI (Arrumação) } & 22 & 23 & 24 & 25 & 26 & 27 & 28 & \multirow{5}{*}{$\begin{array}{c}\text { Fazer } \\
\text { arrumação no } \\
\text { Setor }\end{array}$} \\
\hline & 29 & 30 & 1 & 2 & 3 & 4 & 5 & \\
\hline & 6 & 7 & 8 & 9 & 10 & 11 & 12 & \\
\hline & 13 & 14 & 15 & 16 & 17 & 18 & 19 & \\
\hline & 20 & 21 & 22 & 23 & 24 & 25 & 26 & \\
\hline
\end{tabular}




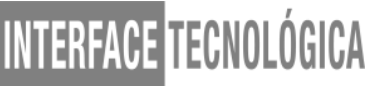

\begin{tabular}{|c|c|c|c|c|c|c|c|c|c|}
\hline & 27 & 28 & 29 & 30 & 31 & 1 & 2 & \\
\hline & & 10 & 11 & 12 & 13 & 14 & 15 & 16 & \\
\hline & & 17 & 18 & 19 & 20 & 21 & 22 & 23 & \\
\hline & & 24 & 25 & 26 & 27 & 28 & 29 & 30 & \\
\hline SEITON & CLASSIFICAÇÃO & 1 & 2 & 3 & 4 & 5 & 6 & 7 & $\begin{array}{c}\text { Classificar } \\
\text { Peças / Maq / } \\
\text { Produtos } \\
\end{array}$ \\
\hline SEISOU & LIMPEZA & 8 & 9 & 10 & 11 & 12 & 13 & 14 & $\begin{array}{l}\text { Limpeza do } \\
\text { Setor }\end{array}$ \\
\hline SEIKETSU & HIGIENE & 15 & 16 & 17 & 18 & 19 & 20 & 21 & $\begin{array}{c}\text { Uniformes / } \\
\text { Cuidado } \\
\text { Pessoal }\end{array}$ \\
\hline SHITSUKE & $\begin{array}{c}\text { AUTO } \\
\text { DISCIPLINA }\end{array}$ & 22 & 23 & 24 & 25 & 26 & 27 & 28 & $\begin{array}{l}\text { Treinar toda } \\
\text { Equipe }\end{array}$ \\
\hline
\end{tabular}

Fonte: Elaborado pelo autor 2018

\subsection{Senso de utilização - no setor}

De acordo com Ribeiro (1994) diz que devemos fazer uma avaliação da utilização daquilo que é necessário do desnecessário como materiais, equipamentos e ferramentas, descartando ou dando a devida destinação àquilo considerado desnecessário ao exercício das atividades. Está definição foi aplicada ao setor de desenvolvimento fazendo reconhecer tudo aquilo que é necessário do desnecessário.

\subsection{Senso de ordenação - no setor}

Este senso apresenta critérios para estocar, guardar ou dispor materiais, equipamentos e ferramentas de modo a facilitar o seu uso e manuseio, facilitar a procura, localização e guarda de qualquer item. Fazer a definição dos locais apropriados, adotando-se como critério a facilidade para estocagem, identificação, manuseio, reposição, retorno ao local de origem após uso, consumo dos itens mais velhos primeiros, dentre outros (VIEIRA FILHO, 2003).

Esta definição é para gerar uma cultura entre os colaboradores do setor a ter hábitos de organização.

\subsection{Senso de limpeza - no setor}

Oliveira (1997) apresenta que senso de limpeza deve fazer a eliminação das sujeiras ou objetos estranhos para manter limpo o ambiente. Sendo que o mais importante no ambiente de trabalho é o limpar, mas o ato de "não sujar". Isto significa que além de limpar é preciso 


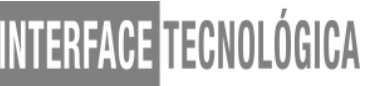

identificar a fonte de sujeira e as respectivas causas, de modo a podermos evitar que isto ocorra.

Esta ferramenta apresentou e implicou em ajustar a rotina dos colaboradores de fazer a limpeza do local a todo momento, garantindo que o ambiente sempre esteja preparado para receber qualquer tipo de serviço.

\subsection{Senso de higiene - no setor}

Trazer condições favoráveis à saúde física e mental, para garantir um ambiente não agressivo e manter boas condições de trabalho; zelar pela higiene pessoal e cuidar para que as informações e comunicados sejam claros, de fácil leitura e compreensão.

Trazer aos colaboradores algo que possam ter comportamento ético, promover um ambiente saudável nas relações interpessoais, sejam sociais ou profissionais, cultivando um clima de respeito mútuo nas diversas relações (VIEIRA FILHO, 2003).

Com o senso de higiene os colaboradores receberam treinamento para que possam manter desde uniformes limpos a EPIs sempre dentro do prazo de validade garantindo a saúde da equipe.

\subsection{Senso de autodisciplina - no setor}

Conforme Oliveira (1997) o senso de autodisciplina é garantir que todas as regras e procedimentos dos outros sensos sejam aplicados de forma eficaz e cause mudanças comportamentais. Este hábito será resultado do exercício da força mental, moral e física.

O importante é garantir que o desenvolvimento resultante do exercício da disciplina inteligente que é a demonstração de respeito de cada um ao programa quando apresentado pela empresa, dentro do setor de desenvolvimento. E os resultados é demostrado com a execução diária de cada colaborador.

\section{PROBLEMAS ENCONTRADOS NO SETOR DE DESENVOLVIMENTO}

Devido a desorganização do setor como:

1. Gabaritos fora de lugar

2. Falta de organização de definição das posições e Lay out 


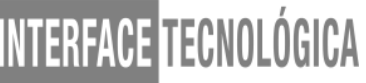

3. Sujeiras espalhadas

4. Maquinas de solda espalhadas pelo setor sem que haja box de solda para produção

5. Maquinas mal posicionadas (furadeiras e maquinas de corte)

6. Colaboradores sem capacitação profissional Ex: senso de organização (programa 5S)

7. Falta de OF (Ordem de fabricação), para controle dos desenvolvimentos de gabaritos / novos produtos / amostras

Mostrado na Ilustração 2.

Ilustração 2: Antes da implantação do programa 5S
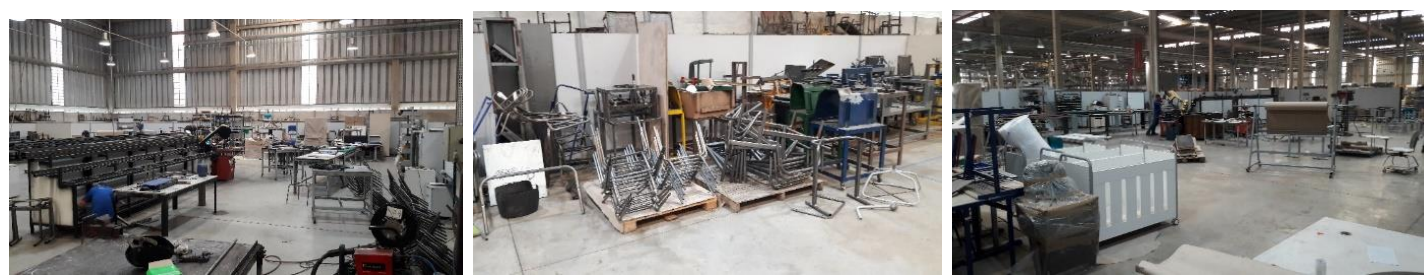

Fonte: Elaborado pelo autor 2018

Assim como notamos nas imagens a como um ambiente desorganizado, como falta de fluxo continuo, sem posicionamento adequado para maquinas, sem identificação no ambiente, e com isso pode se perceber que um produto quando estiver em execução não irá apresentar controle de qualidade relacionado ao trabalho, com prazos estabelecidos.

\section{SUGESTÕES PARA OS PROBLEMAS ENCONTRADOS NO SETOR DE DESENVOLVIMENTO}

$\mathrm{O}$ conceito do programa $5 \mathrm{~S}$ foi apresentado como ferramenta para reestruturar o setor de desenvolvimento desde a organização até a capacitação dos colaboradores, na empresa moveleira.

A empresa tem a necessidade de reestruturar o setor de desenvolvimento aplicando o conceito da ferramenta como a organização, arrumação, limpeza, higiene e autodisciplina. Essa ferramenta se apresenta como base para uma certificação ISO 9001:2015;

A empresa tem por objetivo sempre pensar na eficiência e eficácia de todo trabalho que venha desenvolver, utilizando sempre de sua tecnologia e mão de obra especializada.

Para alcançar o objetivo do programa e obter as Vantagens desejadas como: 


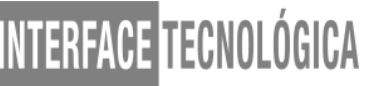

- $\quad$ Aumento da qualidade do produto ou serviço;

- $\quad$ Aumento da produtividade;

- $\quad$ Fornece a base necessária para implementar outros programas de qualidade;

- $\quad$ Facilita a detecção de erros, objetos fora do lugar e outros problemas que precisam de atenção;

- $\quad$ Prevenção de acidentes;

- $\quad$ Melhoria do ambiente de trabalho;

- $\quad$ Melhoria da qualidade de vida;

Todos os envolvidos e todas as mudanças terão que acontecer e respeitar a filosofia do Programa pois se algumas das etapas não entender alguns dos 5 sensos da ferramenta de qualidade, ao invés de buscarmos evolução estaremos andando para trás criando mais problemas do que já existe. O importante é investigar a causa das dificuldades e apresentar as soluções para o crescimento da empresa.

\section{TREINAMENTO E CAPACITAÇÃO}

De acordo com Chiavenato (2010) objetivo do treinamento e desenvolvimento organizacional é mostrar a importância do processo como vantagem para conquista da qualidade dentro de uma organização. Uma organização bem treinada e desenvolvida está apita para manter um alto padrão de qualidade de serviços. Para uma organização, sua principal ferramenta é a mão de obra, então deve estar bem treinada, qualificada e motivada. E para manter esta qualidade como diferencial, ela deve buscar manter o seu quadro de empregados sempre reciclados e treinados.

No setor de desenvolvimento todos os colaboradores receberam treinamento e capacitação do conceito Programa 5S para que possam aplicar no seu dia a dia.

A capacitação se deve por meio de aulas teóricas e aplicação diária do conhecimento adquirido pelo treinamento, e a direção sobre o fluxo de informações que deve acontecer por meio das ordens de fabricação controladas por entradas e saídas, obtendo os registros de cada processo.

Percebe-se que o treinamento pode trazer um grande retorno para o profissional e para a empresa, pois um profissional bem treinado e qualificado terá uma motivação maior e o seu resultado na execução das tarefas será maior e mais produtivo, consequentemente a maior 


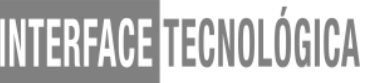

produtividade do empregado poderá contribuir efetivamente para os resultados da organização (ROBBINS, 2002).

Todo setor de protótipo foi sinalizado, por meio de identificações de maquinas, produtos e dispositivos de soldas assim como na Ilustração 3, para que o colaborador após o treinamento pudesse ter alto desempenho.

\section{Ilustração 3 - Sinalização e identificação do ambiente}
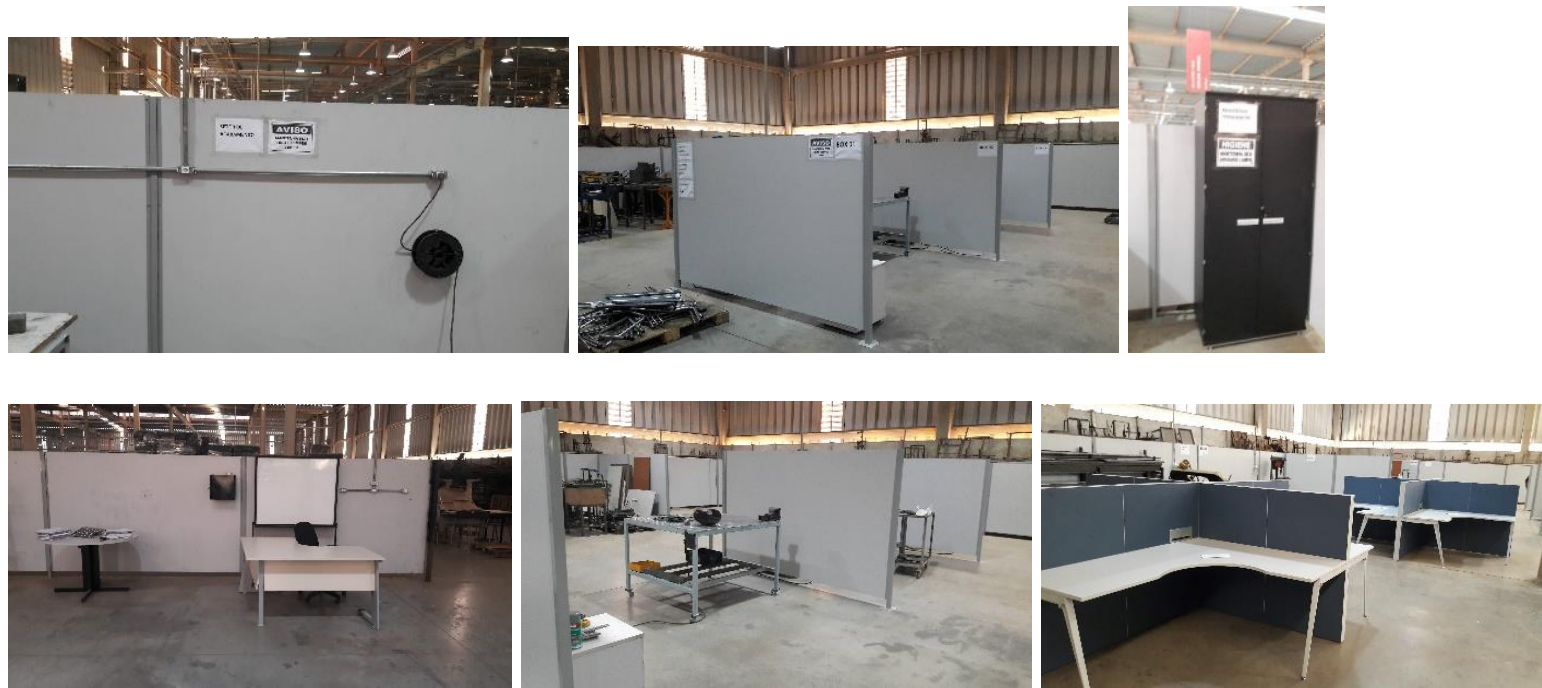

Fonte: Elaborado pelo autor 2018

Com a análise das imagens foi identificado que o conceito de limpeza e organização foram aplicados trazendo um ambiente agradável aos colaboradores.

\section{CONCLUSÃO}

Com a identificação de um setor desorganizado e que apresentava desperdícios de matéria-prima, produtos com custos elevados e atraso na fabricação a empresa moveleira apresentou a necessidade de melhorar a qualidade de seus produtos com prazos estabelecidos e como uma produção de baixo custo.

Com implementação do Programa 5S, a empresa alcançou seu objetivo por meio de treinamentos e capacitação, mudança no lay-out, limpeza, organização e orientações visuais distribuídas no setor de desenvolvimento que impactaram na rotina dos colaboradores da empresa e passaram a valorizar todas as etapas dos sensos: utilização (seiri), ordenação (seiton), limpeza (seiso), saúde (seiketsu) e disciplina (shitsuke). Essa valorização e envolvimento de todos acarretou na eliminação de desperdício de matéria prima, organização e limpeza, fazendo com que cada funcionário faça uma análise crítica de seu trabalho ou do 


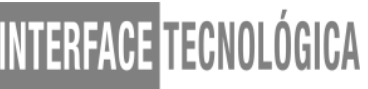

produto em transformação, com a garantia de um ambiente seguro, e com orientações constantes da retomada de cada senso. Os resultados obtidos foram comprovados da confecção dos produtos que apontaram baixo custo, redução no desperdício de matéria prima, redução do custo na fabricação levando a empresa a ser competitiva no mercado e processos licitatórios novamente. E com estes resultados a empresa visa a certificação ISO 9001:2015, com base no conceito do método do programa 5S para toda a empresa.

\section{REFERÊNCIAS}

ABRANTES, J. Programa 8S: Da alta administração à linha de produção: o que fazer para aumentar o lucro? O combate aos desperdícios nas empresas, protegendo o meio ambiente e facilitando o desenvolvimento sustentável. Editora Interciência, Rio de Janeiro, 2007, 2a edição.

CAPERUCCI J. N. Os benefícios do programa 5S em uma Organização, UniSalesiano@, n. 15, julho, 2016. Disponível em: .

<http://www.salesianolins.br/universitaria/artigos/no15/artigo119.pdf> Acesso em: 31 julho 2018

CHIAVENATO, I.; SAPIRO, Gestão de pessoas: o novo papel dos recursos humanos nas organizações. 3. ed. Rio de Janeiro: Elsevier, 2010.

COELHO DA COSTA, C. de O. C. Implantação do método 5S no setor de estoques de um supermercado pertencente a uma rede varejista da cidade de Esperança-PB, VII Congresso Brasileiro de Engenharia de Produção, Ponta Grossa-PR, n. 06 a 08, dez. 2017.

DONIDA, I. C., Implantação do Programa 5S em Empresa de Moda Intima, Centro Universitário Univantes, Novembro, 2014, Disponivel:.< https://www.univates.br/tecnicos/media/artigos/ISABEL_C_DONIDA.pdf> Acesso: 02 setembro 2018

FAVARIN, E. G., CANDIDO, R. R. Estudos dos impactos do programa 5S na empresa point lanches, Fucap, outubro, 2012. Disponível:.< http://www.fucap.edu.br/portal/template/projeto2016/documentos/372e0244d9fb266d5705cc 9e11f6a6a5.pdf > Acesso em: 02 setembro 2018

FILHO, G. V. Gestão da qualidade Total: uma abordagem prática. Alínea Editora, Campinas, SP, 2003.

FLEURY, C. R.; 5S e seus desdobramentos: Uma revisão da literatura, Univ., Goiás, n. 06, set. 2017. Disponível em:

<http://www.unirv.edu.br/conteudos/fckfiles/files/5S\%20E\%20SEUS\%20DESDOBRAMEN TOS\%20UMA\%20REVIS\%C3\%830\%20DA\%20LITERATURA.pdf>Acesso em: 31 jul. 2018

GONZALEZ, E. F. Aplicando 5S na construção civil. Florianópolis: UFSC, 2005. 


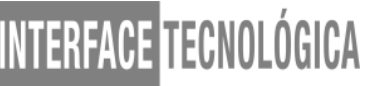

ISHIKAWA, K. Controle de qualidade total à maneira japonesa. Rio de Janeiro. Editora Campus, 1993.

OLIVEIRA, J. R. C. Aspectos humanos dos 5 sensos: uma experiência prática. Rio de Janeiro: Qualitymark, 1997

RIBEIRO, H. 5S: A base para a qualidade total. 10. Ed. Salvador: Casa da qualidade, 1994

RIBEIRO, H. A Bíblia do 5S, da implantação a excelência, Salvador: Casa da Qualidade, 2006.

RIOS, A. I. R. A implementação do programa de qualidade 5s na empresa Wayne Fuelling systems: uma abordagem das atividades dos departamentos de inspeção de recebimento e metrologia, XII Congresso Nacional de Excelência em Gestão, Inovarse@, n. 29 e 30, set. 2016. Disponível em: . <http://www.inovarse.org/sites/default/files/T16_M_019.pdf> Acesso em 31 julho 2018.

ROBBINS, S.P. Comportamento Organizacional. Rio de Janeiro, LTC, 2002.

SCHNEIDER, V., Implantação da Ferramenta de qualidade " $5 \mathrm{~S}$ " em empresa de embalagens de papelão, Centro Universitário Univates, junho, 2015. Disponível:

<https://www.univates.br/tecnicos/media/artigos/vivian.pdf> Acesso em: 02 setembro 2018.

SILVA, J. M. 5S: O ambiente da qualidade. Belo Horizonte: Fundação Christiano Ottoni, 1994.

SLACK, N.; CHAMBERS, S.; JOHNSTON, R. Administração da Produção. Tradução Maria Teresa Corrêa de Oliveira. $3^{\mathrm{a}}$ ed. São Paulo: Atlas, 2009.

SOUSA, C. A. P., OLIVEIRA, G. J., SANTOS, J. G. M., SODRÉ, S. C. D M., MILANI, W. N., A Importância do Treinamento de Pessoal para a Sobrevivência da Organização, Fórum de Pesquisa Cientifica e Tecnológica de Ponte Nova, Disponivel:<

https://even3storage.blob.core.windows.net/anais/74743.pdf> Acesso: 08 setembro 2018.

XAVIER DE LIMA M. A. A aplicação do programa 5s para melhoria da gestão de estoques do setor de almoxarifado de tecidos de uma indústria de confecção, Revista Uningá Review, abril/junho 2018.

ZANELLA, L. C. Programa de qualidade total para empresas de pequeno e médio porte: roteiro prático de implantação. Juruá Editora, Curitiba, 2009 